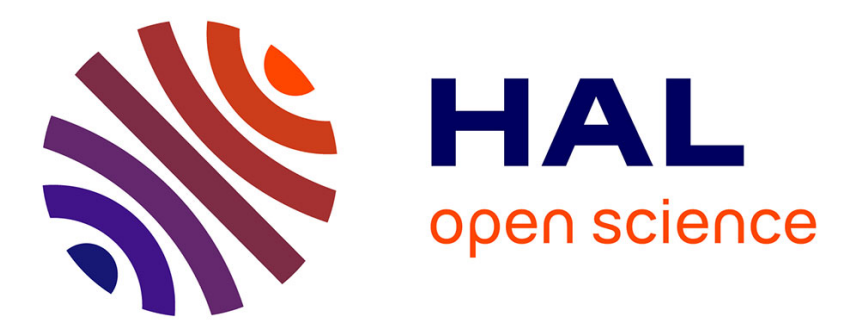

\title{
An Integrated Approach for Value-oriented Energy Forecasting and Data-driven Decision-making. Application to Renewable Energy Trading
}

Thomas Carriere, Georges Kariniotakis

\section{- To cite this version:}

Thomas Carriere, Georges Kariniotakis. An Integrated Approach for Value-oriented Energy Forecasting and Data-driven Decision-making. Application to Renewable Energy Trading. IEEE Transactions on Smart Grid, 2019, 10 (6), pp.6933 - 6944. 10.1109/TSG.2019.2914379 . hal-02124851

\section{HAL Id: hal-02124851 \\ https://hal.science/hal-02124851}

Submitted on 9 May 2019

HAL is a multi-disciplinary open access archive for the deposit and dissemination of scientific research documents, whether they are published or not. The documents may come from teaching and research institutions in France or abroad, or from public or private research centers.
L'archive ouverte pluridisciplinaire HAL, est destinée au dépôt et à la diffusion de documents scientifiques de niveau recherche, publiés ou non, émanant des établissements d'enseignement et de recherche français ou étrangers, des laboratoires publics ou privés. 


\title{
An Integrated Approach for Value-oriented Energy Forecasting and Data-driven Decision-making. Application to Renewable Energy Trading
}

\author{
Thomas Carriere and George Kariniotakis, Senior Member, IEEE
}

\begin{abstract}
Short-term forecasts of generation or demand are required as inputs into several power system management functions. Forecast models are in general tuned to provide optimal accuracy and reliable estimations of associated uncertainty. In a second step, these forecasts are used as input in tools that perform various functions such as scheduling, reserves estimation, and trading in electricity markets. These functions often integrate algorithms that in turn optimize a value-related criterion, like cost, reliability, income, etc. The literature has shown that in some processes, like energy trading, this value may be optimized if a specific quantile forecast is selected rather than the forecast considered as most accurate. In this paper we propose a new data-driven approach in which the two steps of forecasting and decision-making are unified into one function that is optimized considering a single criterion, i.e. the value featured in the decision-making process. This approach allows us to bypass the use of specific forecasting models and could be extended to any decision-making process for which the inputs, outputs and objective functions are well defined. An intermediate approach is evaluated where a meta-optimization is applied to tune the forecast model as a function of the value it brings. This intermediate approach can also prove efficient, but does not allow us to bypass the forecasting models. The applications considered to evaluate the concept are photovoltaic and wind power generation trading in a day-ahead market, where we simultaneously optimize a forecasting model and a trading strategy, considering the final revenue on the electricity market as the objective function.

For the meta-optimization, an Analog Ensemble (AnEn) model is used to forecast PV production, coupled with a Support Vector Regression (SVR) to forecast market prices. For the data-driven approach, we use an Extreme Learning Machine (ELM). As the objective function is highly complex and non-linear, the optimization is carried out by Particle Swarm Optimization (PSO).
\end{abstract}

Index Terms-Data-driven decision making, energy trading, machine learning, price forecasting, renewable energy forecasting

\section{INTRODUCTION}

$\mathbf{T}$ HE increasing share of weather-dependent renewable energies (RES), like wind and photovoltaics (PV), in the global energy mix creates a significant challenge for electricity grid operators. The inherent variability of RES generation can cause mismatches between energy production and expectation. On electricity markets, RES plant operators support this risk by paying for discrepancies. Therefore, obtaining accurate energy

T. Carriere and G. Kariniotakis are with MINES ParisTech, PSL University, Centre PERSEE, CS 10207, rue Claude Daunesse, 06904 Sophia Antipolis Cedex, France (e-mail: thomas.carriere, georges.kariniotakis each with @mines-paristech.fr)

T. Carriere is also with Third Step Energy, 55 allée Pierre Ziller, 06560 Sophia Antipolis Cedex, France (e-mail: thomas.carriere@thirdstep.energy) production forecasts is crucial for effective management of RES power plants. As a result, a wealth of forecasting models have been studied in the literature. Reference [1] provides a thorough review of existing approaches. These forecasts are then used as input for different applications, such as trading in electricity markets, congestion management, scheduling, and reserves estimation [2]. In cases of high RES penetration it is crucial to consider uncertainty in the decision-making process. This involves using probabilistic rather than spot forecasts in the form of probability density function (PDF), quantiles, scenarios or otherwise [3].

Previous works consider forecasting and decision-making as two distinct modeling steps, each optimized based on their own criteria. Forecasting models are built to optimize prediction performance, for example Root Mean Square Error (RMSE) or reliability, and then used as input into the decision-making function, which either just translates into value or is optimized to maximize value. The term "value" refers to the optimization objective of the decision-making process. It may correspond to the reduction of operation costs, the maximization of revenue in a market, etc.

Previous research on forecasting, such as within the European project SafeWind, has concluded that a future perspective involves optimizing forecasting models to take into account not only prediction performance of the models, but also their final use and how this is translated into value through the function that uses these forecasts as input for decision-making. However, the value of a given forecast is different for different users e.g. energy producers or Transmission System Operators (TSO). In reference [4], the authors showed that a wind power plant operator could benefit more from biased forecasts when trading on an electricity market, while the TSO would benefit more from an unbiased forecasting model. This was illustrated in [5] where an analysis of how the bids submitted in an electricity market changed with respect to the objective function of the decision-making model. However, the different objective functions were not also used to tune the wind power forecasting model, but only the trading unit. Reference [3] suggested that in uncertainty forecasts, end-users should use different uncertainty representations depending on the final application. It also provided guidance on which forecast properties are preferable for five typical applications of wind power forecasting. Similarly, [6] proposed new evaluation frameworks for solar power forecasting that incorporate the economical value of the forecasts.

Following these works, the motivation behind this paper 
is to address this perspective, thus making a radical change to the classic paradigm by developing a closer link between the two steps of the standard modeling chain, i.e. forecasting and decision-making. More precisely we propose a new datadriven approach in which the two steps of forecasting and decision-making are unified into one function that is optimized considering a single criterion, i.e. the value featured in the decision-making process. This approach can bypass the development of specific forecasting models and can be generalized to any decision-making process for which the inputs, outputs and objective functions are well defined. An intermediate approach is evaluated, where a meta-optimization is applied to tune the forecast model as a function of the value it brings, however this is not generalizable since it uses specific models depending on the decision-making process considered.

The proposed fully data-driven approach may be potentially applicable to various types of complex problems. An example is the problem of a virtual power plant participating in energy and ancillary services markets, i.e. as in [19]. The forecasting step involves forecasting models for renewable generation and 8 market quantities and a model for decision-making. In total, 10 models need to be built, tuned and maintained operationally. Following the approach proposed here, they can be replaced by a single model. The data-driven approach can be suitable for implementation in automated applications like trading agents. It simplifies the overall modeling chain and thus reduces maintenance needs and the propagation of errors. In order to demonstrate the proof of concept, in this paper we narrow the area of application to the trading of RES generation, namely PV and wind production, on a day-ahead energy market. The original modeling chain in this problem involves five steps (PV forecasting, price forecasting, positive and negative imbalance cost forecasting and the trading algorithm).

Reference [7] linked the predictability of a power plant to its revenue. However, the goal was to arbitrate between predictability and the capacity factor of wind power plants at the investment phase. Numerous papers have proposed advanced trading models to participate in the energy market [8], [9] and used the revenue generated as a measure of the performance of their work. However, these approaches were developed in an open-loop setup, and although the performance in terms of income was assessed, it was not fed back to optimize the structural parameters of the forecasting or the trading model itself. On the other hand, reference [10] used a reinforcement learning approach to make bids on an electricity market based only on market data, without knowing the exact market rules. Nevertheless, a forecast of the renewable power generation was required, and the forecasting and decision-making steps were two separate parts of the modeling chain.

The key contribution of this paper is to propose two approaches that link the two steps of forecasting and decisionmaking. In a first approach, we introduce a meta-optimization of the forecast models, where all of the hyperparameters from the models involved in the decision-making process are optimized at once. The objective function we propose to perform this optimization is the value generated from the model forecasts with the decision-making (trading) process.
The second approach goes further by merging the forecasting and decision-making steps into a single modeling step where the input is potentially composed of the inputs of the initial models and the output is the output of the decision-making process. This is a fully data-driven decision-making approach, as explained earlier.

Both approaches require optimization loops that use the revenue generated on the electricity market as the objective function. Such optimizations can be difficult to perform, as the loss function has to be evaluated using PV power forecasts over a significant time period, and is nonlinear. As a result, a heuristic optimization algorithm is employed. Popular heuristics include Genetic Algorithms (GA) [11], or Particle Swarm Optimization (PSO) [12]. Reference [13] suggests that the PSO algorithm is generally better in terms of convergence, but that it has a higher computational cost because of the communication required between the particles for each generation. However, since the function evaluations are quite long, this communication cost is low compared to the evaluations. Thus, we use the PSO algorithm as an optimization engine.

Both approaches are compared with the standard two-step approach in the literature through two case-studies featuring real-world data from PV plants in France with the EPEX dayahead market, or wind farms in Denmark with the NordPool day-ahead market. For probabilistic PV power forecasting, we use a model from the Analog Ensemble (AnEn) family with accuracy comparable to the state-of-the-art [14], [15], [16]. The AnEn model relies on k-nearest-neighbor ( $\mathrm{kNN})$ methodology, which is well understood [17], so that its behavior can be controlled through its hyperparameters. Wind power forecasts come from a previous study [18]. All simulations were performed with the software $R$, using the additional packages $e 1071$ [19] and pso [20]. They were partially parallelized and run on a $2.5 \mathrm{GHz}$ quad-core CPU with $16 \mathrm{~GB}$ of RAM.

In general, advanced trading of RES production on dayahead markets involves both RES generation forecasts and forecasts of day-ahead and regulation prices [21], [22], [23]. Regarding the price forecasts, a review of price forecasting models is proposed in [24]. Here, we use a standard Support Vector Regression (SVR) approach for price forecasts, as they are only used to test the methodology. For trading on multiple markets, like ancillary services, the trading process may also involve forecasting several additional quantities (i.e. in [25]). The model chain inevitably becomes very complex in these cases.

The paper is organized as follows. Section II presents the different approaches that we will compare in the following sections. Section III presents the first test case on which these approaches are compared, and the forecasting tools used to implement these approaches. Section IV presents the results obtained on the second test case. Finally, section III-F analyzes the results from the test case and section $\mathrm{V}$ draws the conclusions of the paper.

\section{Methodology of the Different Approaches}

In this work, we propose two alternative approaches to the standard implementations of decision-making processes 
in energy trading. Usually, these standard implementations require numerous models specific to the given process. The output of these models is combined following a strategy to derive the optimal decision. For example, in the case of RES energy trading in a dual-price market, four models are required: one for forecasting the RES production, one for the day-ahead spot price, and two for the imbalance prices for positive and negative imbalances.

As mentioned above, each of the dedicated forecast models contains meta-parameters. Typically in the literature, each model is optimized for its own performance ("Reference" approach hereafter). In the first approach we propose here, we jointly and simultaneously optimize the meta-parameters of all three types of forecast models under a common objective. All of the approaches are represented on Fig. 1.

To formalize the different approaches, we consider an application where a decision $D(X)$ has to be taken conditionally to a set of inputs $X$. We suppose that the standard approach is given by a trading strategy applied to a combination of different dedicated forecasting models using the inputs $X$. We note as $M_{1, \ldots, n}(X)$ each of the dedicated models forecasting the quantities $y_{i}$ involved in the decision process, and as $T\left(y_{1}, \ldots, y_{n}\right)$ the trading strategy that combines the outputs to obtain the optimal decision. The decision-making process is thus modeled by:

$$
D(X)=T\left(M_{1}(X), \ldots, M_{n}(X)\right)
$$

Furthermore, we consider that each of the $n$ dedicated models uses a set of parameters $\Theta_{1, \ldots, n}$, and we note as $M_{i}^{\Theta_{i}}$ the output of the $i$-th model using the parameters $\Theta_{i}$. These parameters define the decision-making process entirely, and are calculated differently in the different approaches.

Finally, we suppose that we have a function $\operatorname{Eval}(d)$ that evaluates the value associated with the decision $d$. Based on these generic notations, we formulate hereafter the three approaches that we will compare.

\section{A. Reference Approach: Separate Optimization}

The reference approach consists in separately optimizing the PV power forecasting model and the three models that predict the market quantities. This is the most intuitive approach, as it seems natural that the whole trading chain would perform better if the specific performance of each element of the chain were optimized.

To assess the performance of each element, we use the RMSE for deterministic quantities and the Continuous Ranked Probability Score (CRPS) for probabilistic quantities. Considering that we have a set of $m$ point forecasts $\hat{y}^{i}$ or cumulative distribution function forecasts $\hat{F}_{y}{ }^{i}, i \in[1: m]$, along with their verification values $y^{i}$, these criteria are defined as follows:

$$
\begin{aligned}
\operatorname{RMSE}(\hat{y}, y) & =\frac{1}{m} \sqrt{\sum_{i=1}^{m}\left(y^{i}-\hat{y}^{i}\right)^{2}} \\
\operatorname{CRPS}\left(\hat{F}_{y}, y\right) & =\frac{1}{m} \sum_{i=1}^{m} \int_{-\infty}^{\infty}\left(\hat{F}_{y}^{i}(x)-\mathbb{1}\left(x>y^{i}\right)\right) d x
\end{aligned}
$$

where $\mathbb{1}()$ outputs 1 if the condition in parenthesis is met, and 0 otherwise. The optimization of the parameters of the whole chain is then performed by identifying the optimums separately. We note the evaluation function $G$, which is the RMSE if the evaluated model is deterministic, and the CRPS otherwise.

$$
\Theta_{i}^{*}=\operatorname{argmin}_{\theta}\left\{G\left(M_{i}^{\theta}(X), Y_{i}\right)\right\}
$$

\section{B. First Proposed Approach: Simultaneous Optimization}

In this approach, we optimize the whole chain globally, using the value of the decision process as the objective function. The point of this optimization is that the individual forecasting models could adapt to each other's forecast errors. For example, if a given model is biased, another model could also acquire a bias to compensate the former. The adaptations that the models could perform on each other are highly dependent on the final objective of the decision process.

Noting as $\Theta=\left\{\Theta_{1}, \ldots, \Theta_{n}\right\}$ the set of parameters that gathers all of the parameters of the individual models, the optimization problem we must solve in this approach is thus:

$$
\begin{aligned}
\Theta^{*} & =\operatorname{argmax}_{\Theta=\left[\Theta_{i}, \ldots, \Theta_{n}\right]}\{\operatorname{Eval}(D(X))\} \\
& =\operatorname{argmax}_{\Theta=\left[\Theta_{i}, \ldots, \Theta_{n}\right]}\left\{\operatorname{Eval}\left(T\left(M_{1}^{\Theta_{1}}(X), \ldots, M_{n}^{\Theta_{n}}(X)\right)\right)\right\}
\end{aligned}
$$

\section{Second Proposed Approach: Direct Bidding with a Unique Model}

The second approach we propose is to bypass the forecasting models, and replace them with a unique model that directly provides the decision, as shown in Fig. 3. The inputs and outputs of this unique model are the same as the inputs and outputs of the global decision-making process.

For each test case, the model we use to provide the decision is an Artificial Neural Network (ANN). One reason for this choice is that ANNs are very sensitive to the tuning of the parameters, since it is the parameters that identify the interaction between the different sources of data.

Models from the ANN family are generally trained using the backpropagation algorithm. This algorithm makes the training of the network very efficient. However, it can only be used when the objective function of the network is differentiable. This is not the case here, since the network is trained using an arbitrary function to derive the value of the decisions. As a result, we use the PSO to train the network. Since we cannot benefit from the efficiency of the backpropagation algorithm, we use the Extreme Learning Machine (ELM) variant of the standard ANN to accelerate the training. ELM consists in 


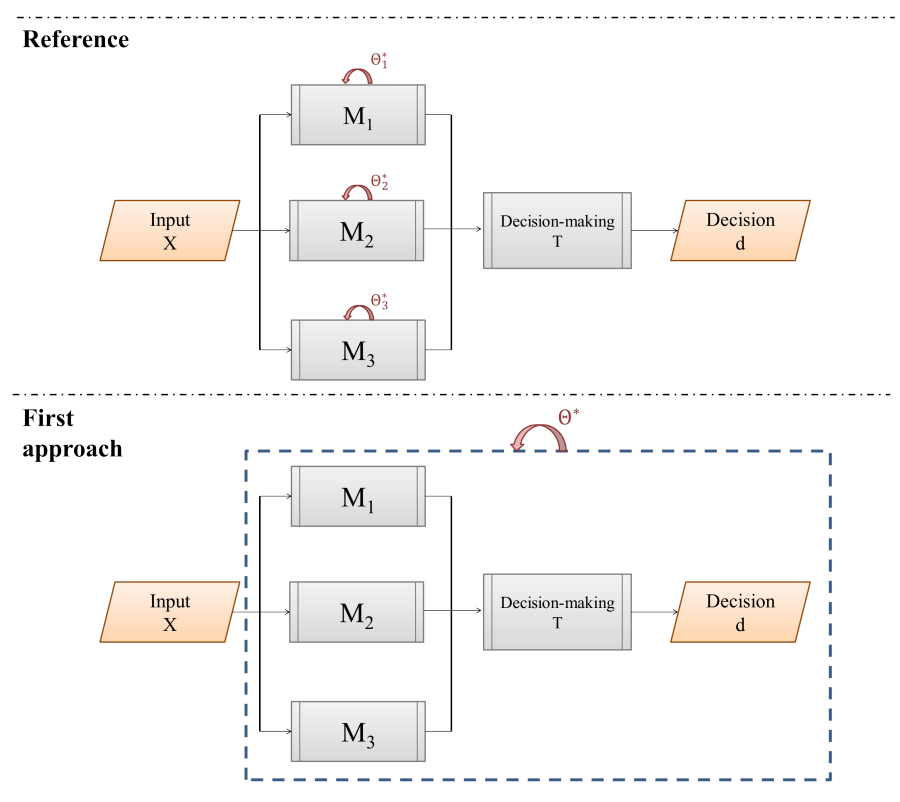

Second

approach

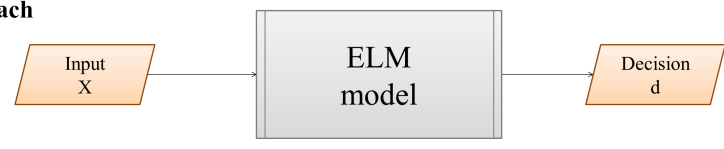

Fig. 1. Schematic representation of the different approaches

randomly setting the parameters of the model except for the output layer, then optimizing the parameters of the output layer only. Training of these networks is very fast, and in addition they are able to approximate any non-linear function [26]. They have also been used to forecast PV power in [12], [27].

The main risk with this model is overfitting. Since the number of parameters is significant, the model can easily memorize the entries from the training set to achieve high accuracy on the training without developing the ability to generalize. However, finely controlling the behavior of the model through the parameters can help the model capture more interaction between the PV-related and market-related inputs.

\section{Test Case I: Trading of PV Power on the EpeX SPOT MARKET}

In this test case, we study the participation of an RES power plant as a Balance Responsible Party (BRP) on a dual-pricing electricity market. The market structure assumed is:

- A day-ahead market where each participant has to submit buying or selling orders the day before delivery.

- A balancing market where each BRP has to take responsibility for its imbalances.

These two markets are sequential. First, the producer submits its bids for the next day. Then after delivery, the producer, which is also a BRP, pays the TSO for its imbalances through the balancing market. Intra-day markets to correct the producers position during the delivery day are not considered.

A dual-pricing electricity market refers to the case where imbalances are settled through two different prices depending on their sign. Usually, if the producer produces more energy than it has sold, then the excess energy is sold at a price lower than the spot prices for this market time unit. On the contrary, when the producer produces less than it has sold, it has to buy the lacking energy at a price higher than the spot price for this market time unit.

Under such markets, the revenue of a producer for a given market time unit is:

$$
R\left(E_{C}\right)=\pi E_{C}+\left\{\begin{array}{l}
\pi^{+}\left(E-E_{C}\right) \text { if } E>E_{C} \\
\pi^{-}\left(E-E_{C}\right) \text { if } E<E_{C}
\end{array}\right.
$$

where $E_{C}$ is the energy sold by the producer, $E$ is the actual delivered energy, $\pi$ is the spot price that is given by the market clearing after the bids from all market participants have been submitted, and $\pi^{+}$and $\pi^{-}$are the regulation prices for positive and negative imbalances, computed by the TSO, depending on the cost it had to meet to compensate the producers imbalances. The price for positive imbalances is usually lower than or equal to the spot price, so that any surplus energy compared to the bids is rewarded at a price lower than the spot price. Similarly, the price for negative imbalances is usually higher than or equal to the spot price, so that any missing energy is penalized at a higher price than the spot price.

In the first approach, we keep the standard model chain for RES energy trading on electricity markets, where RES power and market quantity forecasts are first produced, then used by a trading strategy that estimates the optimal bids. However, we propose a global optimization loop that optimizes the whole model chain using the revenue generated on the electricity market as the objective function. This approach is compared to the reference approach, where we optimize the models separately to maximize their performance, then inject their forecasts into the trading strategy. We will first present the trading strategy, then the RES power and market quantity forecasting models, before formulating the optimization problem we propose to solve with this approach.

\section{A. Trading Strategy}

The strategy we apply is described in [21]. It relies on the hypothesis that the RES producer bids have no influence on the spot price ("price taker" hypothesis). This hypothesis is true when the penetration of price takers is low. However, since increasing numbers of RESs are participating in electricity markets, usually following a price-taker strategy, the influence of RES bids on the spot price can become significant. However, we do not consider this influence in the rest of this article to avoid modeling it.

Based on equation (6), we can rewrite the revenue of a producer as follows:

$$
\begin{aligned}
R\left(E_{C}\right) & =\pi E+\pi_{R}\left(E-E_{C}\right) \\
\pi_{R} & =\left\{\begin{array}{l}
\pi^{+}-\pi \text { if } E>E_{C} \\
\pi^{-}-\pi \text { if } E<E_{C}
\end{array}\right.
\end{aligned}
$$

With this formulation, we can see that the first term in (7) is independent from the bid, as the price-taker hypothesis states 
that $\pi$ is independent from $E_{C}$, and so is the actual energy produced $E$. Note that by definition of the regulation prices, the second term is always negative. Thus, maximizing the revenue is equivalent to minimizing the function $g_{E}\left(E_{C}\right)=$ $\pi_{R}\left(E-E_{C}\right)$. The optimization problem that gives the optimal bid $E_{C}^{*}$ is then:

$$
\begin{aligned}
E_{C}^{*} & =\operatorname{argmin}_{E_{C} \in\left[0, E_{n}\right]}\left\{\mathbb{E}\left[g_{E}\left(E_{C}\right)\right]\right\} \\
& =\operatorname{argmin}_{E_{C} \in\left[0, E_{n}\right]}\left\{\int_{0}^{E_{n}} g_{x}\left(E_{C}\right) f_{E}(x) d x\right\}
\end{aligned}
$$

where $E_{n}$ is the maximum amount of energy that the plant can produce during a market timestep, and $f_{E}$ is the PDF of the actual energy generation $E$. We set $E_{n}$ to the energy quantity that can be produced with the installed power during the length of a market time unit. Replacing $g_{E}\left(E_{C}\right)$ by its actual value, we obtain:

$$
E_{C}^{*}=\operatorname{argmin}_{E_{C} \in\left[0, E_{n}\right]}\left\{\int_{0}^{E_{n}} \pi_{R}\left(x-E_{C}\right) f_{E}(x) d x\right\}
$$

The exact solution to this problem is given by [21]:

$$
E_{C}(t)^{*}=F_{E}^{-1}\left(\frac{\pi-\pi^{+}}{\pi^{-}-\pi^{+}}\right)
$$

where $F_{E}$ is a forecast Cumulative Distribution Function (CDF) of the energy production of the plant. The application of this strategy for an RES power plant then requires input from a probabilistic RES power forecasting model, and from spot and regulation price forecasting models.

\section{B. PV Power Forecasting Model: the Analog Ensemble}

The model we use to produce PV production forecasts for the first case study is an improvement of the AnEn model [14], which is presented in [28]. One advantage of this model is that it is very close to a $\mathrm{kNN}$ estimator, which makes it simple to understand. As a result we can define hyperparameters that control the properties of the forecasts quite well.

The AnEn model performs a search to identify the past situations most similar to the one we want to forecast, based on an adaptive metric on the predictor variables, to create a set of likely values called the Analog Ensemble. The density is then estimated using this set of likely values. In our case, density estimation is performed by a Kernel Density Estimation (KDE) using an Epanechnikov kernel [29].

The metric used to search for the most similar historical situations is a weighted sum of the average Minkowski distance of order $p$ for each feature, on the $k$ time steps preceding the historical instant for which the similarity is assessed. The weights are defined by the mutual information (MI) between the considered feature and the PV generation, conditional to the forecast horizon. These are then normalized so that they add up to 1, using a parameter $q$ to control the ratio between the contribution of the most informative feature and the least informative to the metric.
The MI is often used in machine learning for feature selection [30]. It measures the extent to which the fact of knowing a variable reduces the uncertainty of another variable. The MI between two random variables $X$ and $Y$, knowing their respective marginal density distributions $p_{X}$ and $p_{Y}$ and their joint density distribution $p_{X, Y}$, is:

$$
M I(X, Y)=\iint p_{X, Y}(x, y) \log \left(\frac{p_{X, Y}(x, y)}{p_{X}(x) p_{Y}(y)}\right) d x d y
$$

To calculate the metric between the instant to forecast $t$ and an instant in the past $t^{\prime}$ with a forecast horizon $h$ (that is, if the model is run at $t-h$ ), the metric writes:

$$
\begin{aligned}
\left(\mathbf{X}_{t}, \mathbf{H}_{t^{\prime}}\right) & =\sum_{i=1}^{N_{v}} w_{i}^{h} \sqrt{\sum_{j=-k}^{0}\left(\mathbf{X}_{i, t+j}-\mathbf{H}_{i, t^{\prime}+j}\right)^{2}} \\
i n f_{i}^{h} & =M I\left(L^{h}\left(H_{i}\right), E_{P V}\right) \\
w_{i}^{h} & =\frac{\left(i n f_{i}^{h}\right)^{q}}{\sum\left(\left(i n f_{i}^{h}\right)^{q}\right)}
\end{aligned}
$$

The two sets $\mathbf{X}$ and $\mathbf{H}$ contain respectively the features of the instant to forecast and the historical instants. $N_{v}$ is the number of features used for computing the metric. $L^{h}$ is the lag operator and $E_{P V}$ is the PV electricity generation. Finally, the mutual information $M I$ is computed using empirical estimates of the joint distributions. The variables used to compute the metric are irradiance, temperature, wind speed, cloud cover and humidity forecasts obtained from the European Center for Medium-Range Weather Forecasts (ECMWF). We use also a clear-sky profile computed by the McClear model [31].

The parameters introduced here are the hyperparameters that control the behavior of the metric. $p$ penalizes significant differences between the inputs, while $q$ controls the relative importance of each feature. $k$ is introduced so that an instant $t$ in the evening (resp. morning) cannot be considered similar to an instant $t^{\prime}$ in the evening (resp. morning).

Finally, two remaining parameters allow us to control the bias of the model. The first is the number of most similar historical situations $N$ that we retain for estimating the PDF, and the second is the length $L_{P V}$ of the period preceding the instant to be forecast that we use to search for analogs.

According to our results, the higher these parameters, the lower the bias of the model. When the time period over which the analogs are searched increases, the model loses some of its conditionality on recent weather conditions, since old data is used. In addition, increasing the number of analogs also has the effect that the models conditionality on the actual weather forecasts is lost, because as the number of analogs increases, each additional analog used is observed in a situation that is less similar to the actual weather forecasts. Ultimately, as these parameters increase in number, the more the model tends to produce an unconditional climatological average of the power as a forecast, which would be perfectly reliable, and thus have no bias.

Some illustrative probabilistic forecasts for a given plant are reported on Fig. 2 along with a quantification of the 
TABLE I

Bias of THE MOdels For VARYing $N$ AND $L_{P V}$ VALUeS

\begin{tabular}{cc}
\hline \hline & Bias (\%) \\
\hline$N=240, L_{P V}=50$ & 0.15 \\
$N=240, L_{P V}=150$ & 0.09 \\
$N=720, L_{P V}=50$ & 0.03 \\
\hline \hline
\end{tabular}

models bias in Table I with varying $N$ and $L_{P V}$ values to illustrate this effect. To isolate the effect of these parameters, all the other parameters keep the same value Each shade of grey represents a prediction interval (PI), with increasing steps of $2 \%$. Using a longer time period to identify the analogs, or increasing the number of analogs, results in a curve that looks more like the typical "bell" curve of PV production, neglecting the intra-day variability. Ultimately, when taking the expectancy of the forecast distributions as deterministic estimates of the production, this results in a less biased model in the deterministic sense.

\section{Forecasting Market Quantities}

At the time of the bids, we are not yet aware of the three prices $\pi, \pi^{+}$and $\pi^{-}$. Therefore, we have to implement a forecasting model for these three market quantities.

We use Support Vector Regression (SVR) to obtain a deterministic estimate of the spot price, using as inputs the day of the week, time of day, a forecast of the national energy demand provided by the TSO, and the spot price observed the day before at the same time. SVR models are a machine learning technique commonly used for energy price forecasting [24]. The SVR model uses a radial kernel with a parameter $\gamma$ and we use a parameter $C$ to penalize the constraint violations.

The other prices $\pi^{+}$and $\pi^{-}$are forecast by employing a simple kNN estimator, using the predicted spot price as a feature. The only parameters we use are then $n$, the number of neighbors we retain, and $L_{\text {Price }}$ the length of the period over which we look for neighbors. Depending on whether these parameters relate to the positive or negative regulation price-forecasting model, they are noted $n_{+}, L_{\mathrm{Price}_{+}}$or $n_{-}$, $L_{\text {Price }_{-}}$. As for the AnEn model, these parameters allow us to control the bias-variance trade-off of the model.

\section{Application of the Approaches}

We study the output from the two proposed approaches relating to thirteen PV power plants located in France. Insitu measurements of power injected into the grid, local temperature, and solar irradiance are available for the three plants from May 2015 to May 2017. Weather forecasts from the ECMWF were obtained for the same period. The period from May 2015 to May 2016 was used as a training set and the period from June 2016 to May 2017 as the testing set.

In this test case, following the notations from Section II, we have four individual models:

- $M_{1}^{\Theta_{1}}$, which forecasts the PV power with the parameters $\Theta_{1}=\left[p, q, k, N, L_{P V}\right]$

- $M_{2}^{\Theta_{2}}$, which forecasts the spot price with the parameters $\Theta_{2}=[C, \gamma]$
- $M_{3}^{\Theta_{3}}$, which forecasts the balancing price for negative errors with the parameters $\Theta_{3}=\left[n_{-}, L_{\mathrm{Price}_{-}}\right]$

- $M_{4}^{\Theta_{4}}$, which forecasts the balancing price for positive errors with the parameters $\Theta_{4}=\left[n_{+}, L_{\text {Price }_{+}}\right]$

Following the methodology from Section II, the implementation of the three approaches is straightforward. We also compare the three approaches with an even simpler one, where the bids on the electricity market are simply the expectation of the PV power generation. All of the approaches compared are represented on Fig. 3.

The different approaches are noted "Benchmark" for the base case where the bids are the expectancy of the forecast PV power, "Reference" for the case where each of the 4 models is optimized separately, "Approach 1" for the joint optimization of meta-parameters of the 4 models, and "Approach 2" for the second proposed approach of the single data-driven model.

For the reference and first approaches, the model chain is optimized by solving the optimization problems formulated in Section II over the training set. Then, the optimal chain is used to obtain bids for the testing set. For the second approach, the number of neurons for the ELM network is calculated using a 10 -fold cross validation on the training set. Once the architecture of the ELM is established, it is trained over the whole training period, then used on the testing set to obtain the bids.

Fig. 4 shows the results from the different approaches for a given trading day. These days are randomly chosen for illustrative purposes. The ELM approach is much more conservative than the other ones. This results in a negative bias that will be studied in Section III-F. Besides, it is interesting to note that the reference and first approaches, which use a PV power forecast model, tend to produce bids that are more volatile, while the second approach tends to produce smoother bids. Finally, while the reference and first approaches both use market information and thus do not only rely on PV power forecasts, the second approach proposes bids that deviate comparatively more from the PV power expectancy, because of its revenue-focused optimization.

\section{E. Convergence History and Computational Cost}

Fig. 5 reports the convergence history of our proposed approaches 1 and 2. The training error is monitored and at the point that it seems to stop decreasing, training is stopped. This occurs at around 200 iterations for the second approach, and at only 10 iterations for the first approach.

Parameters tested during the evaluation are saved and evaluated a posteriori on the testing set, to check for any signs of overfitting. The different models do not seem to have overfitted the data, as the testing error does not increase before the end of the training.

The computational costs of each approach are reported in Table II. The training time indicates the time required to perform one iteration of the PSO algorithm for the given approach. The evaluation time is the time required to compute the model over the testing set. Since the learning of the model is delayed until a forecast is requested for the approaches involving the AnEn model (Benchmark, Reference and approach $1)$, the evaluation time is much longer. 


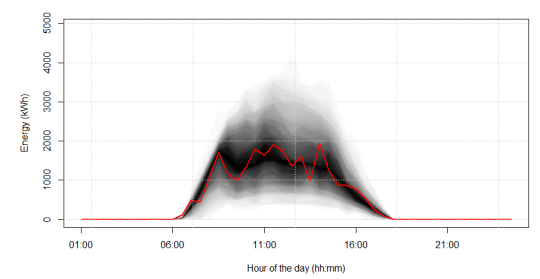

(a) $N=240$ analogs, $L_{P V}=50$ days

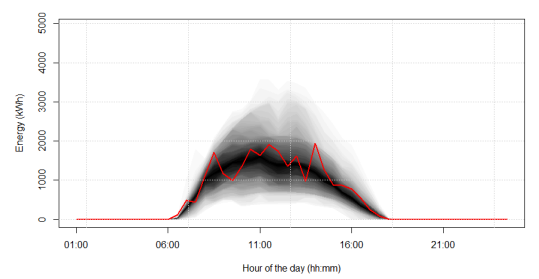

(b) $N=240$ analogs, $L_{P V}=150$ days

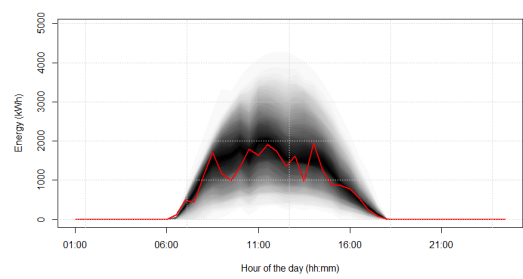

(c) $N=720$ analogs, $L_{P V}=50$ days

Fig. 2. Example of PV probabilistic forecasts for a given day

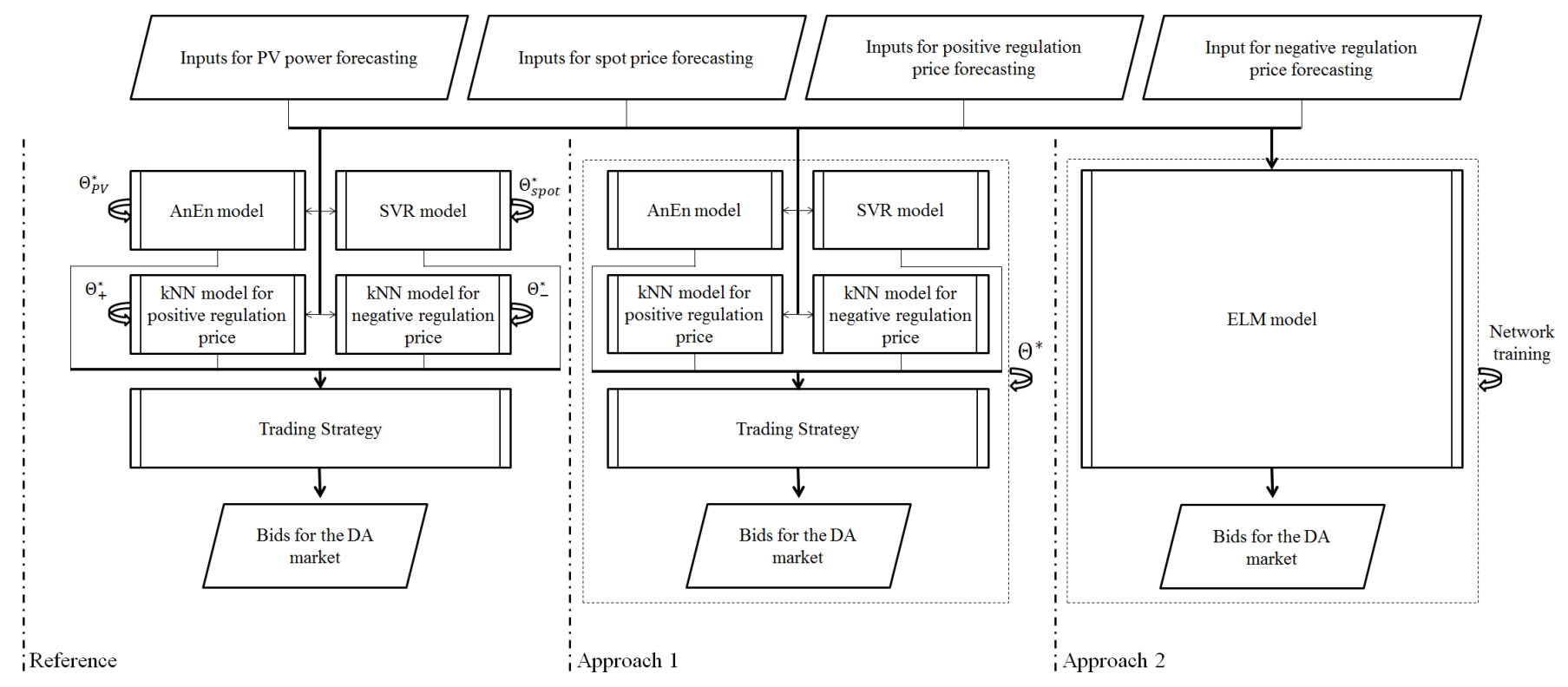

Fig. 3. Flowchart of the different approaches

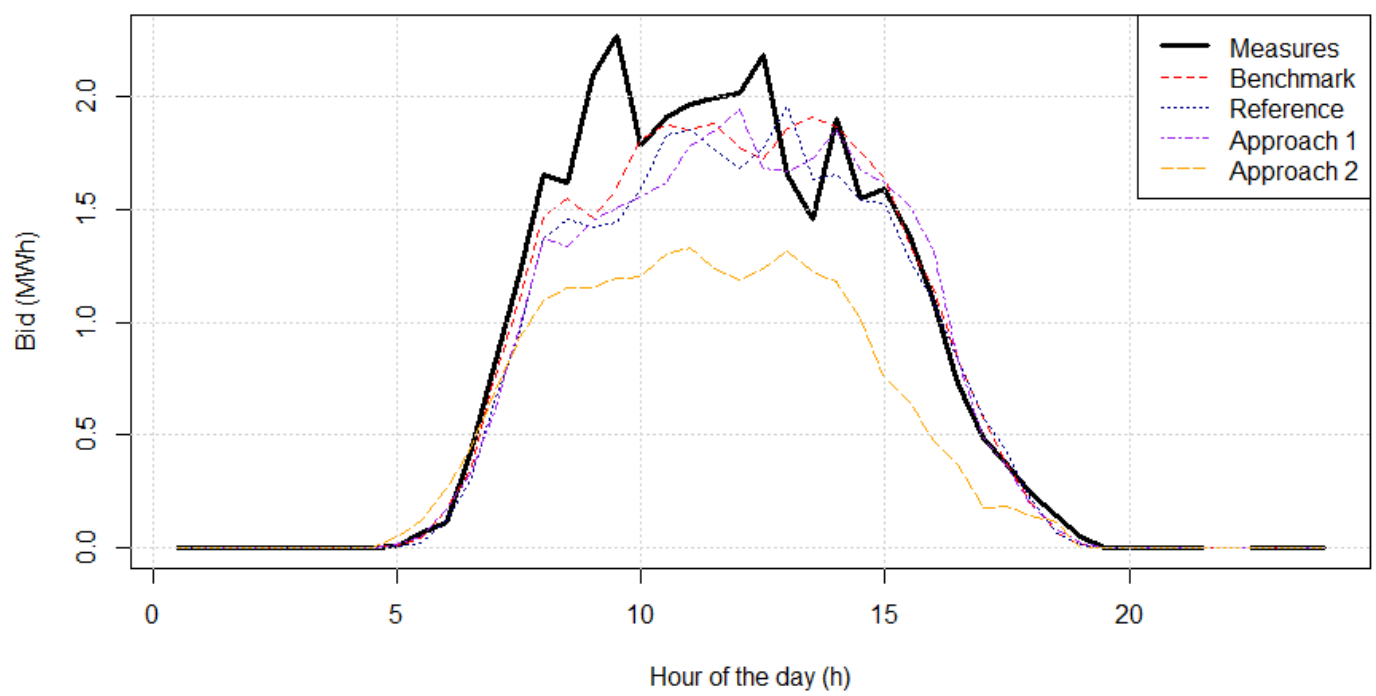

Fig. 4. Bids from the different approaches for day 2016-05-16 


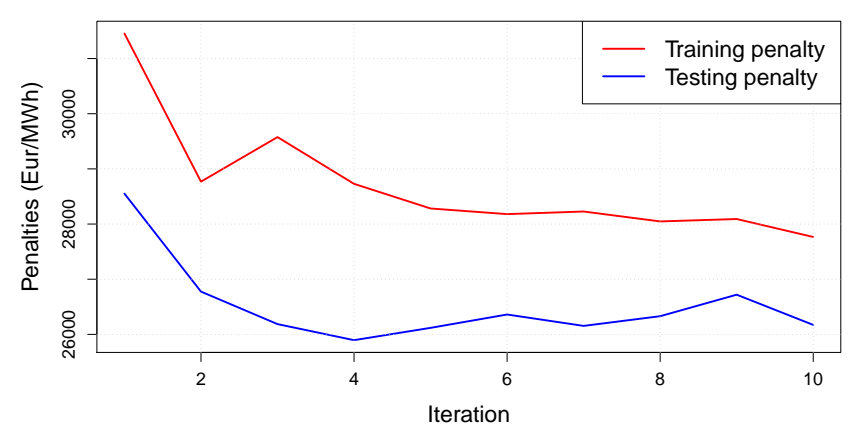

(a) Approach 1

Fig. 5. Convergence history for approaches 1 and 2

TABLE II

Computational Cost of the Different Approaches

\begin{tabular}{ccccc}
\hline \hline & AnEn & SVR & App. 1 & App 2 \\
\hline Training time (s) & 9960 & 1800 & 12740 & 26.4 \\
Evaluation time (s) & 830 & 150 & 980 & 0.78 \\
\hline \hline
\end{tabular}

\section{F. Evaluation Results}

1) Evaluation of the trading strategies: The performances of the different strategies for the different plants are compared on Fig. 6. They show the improvement of each approach for different criteria, relative to the worst performing approach for that plant. Complete quantitative results are reported in Appendix A. The strategy that yields the best revenue varies considerably between all of the plants studied, although all approaches seem to yield very similar revenues.

Of the 13 other plants, none obtained the best revenue with the benchmark approach, 3 with the reference approach, 4 with the first approach, and 5 with the second approach. No approach stands out significantly from the others in terms of net revenue. Still, the first and second approaches produced the best results for 9 out of 13 plants, which suggests that it is worth using revenue as the objective function. It is interesting to note that the models that were optimized with the first approach performed worse according to CRPS and RMSE evaluations, but still generated more revenue.

For all the approaches, a non-convex optimization has to be solved, since all the objective function are non-convex with respect to the model parameters. However, for the 4 plants that produced a higher revenue with the reference or benchmark approach, it is likely that the more complex optimization problems involved in the first and second approaches, which use a more complex function in the final step (the revenue instead of the convex RMSE/CRPS), and more design variables, are more likely to fall within a local minimum that ultimately results in lower revenues.

However, what is interesting is that the second approach systematically causes the highest imbalances, yet produces the lowest penalty per imbalance. Thus, it seems that the ELM model involved in this approach focuses more on reducing

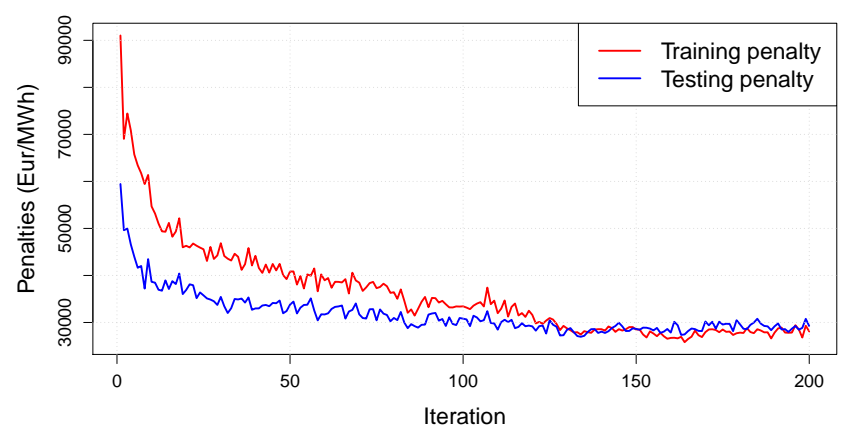

(b) Approach 2

errors when the regulation prices are significant than on reducing the total amount of errors.

More generally, the more market information the approaches include, the more imbalances they generate, but with fewer penalties per imbalance. The reference approach generates a low error but a high penalty per imbalance. When using approach 1 instead of the reference approach, and thus incorporating market information in the PV power forecasting model, the imbalances increase but the penalties per imbalance decrease. Finally, the second approach results in the most imbalances but the lowest penalties per imbalance.

2) Behavior of trading strategies: To understand the reason why the different approaches perform differently, we analyze the bidding behavior of the different approaches. First, we analyze the bias in the bids to understand how the different approaches use the market information. Table III shows the average bias of the approaches over all of the plants.

From the bids error statistics, we can see that adding market information to PV power forecasts without considering the value creates a significant bias, because the bids are not the expected outcome of the distribution, but a given quantile that depends on market information. Depending on how often positive or negative errors are penalized, the bids can then show a tendency to prefer positive or negative errors. However, it seems from the results that the market information that was used was not sufficiently accurate that using it without other considerations would increase revenue. Taking the first approach, the bids bias decreases, to the level of the benchmark approach. Still, the revenue is higher. This suggests that taking the first approach allows the models to use market information in order to increase the revenue without abandoning the bias.

For the second approach however, the bids acquire a significant negative bias. This can be explained by the magnitude of the regulation prices. Over the period of the test case, the negative errors were penalized by $21.2 € / \mathrm{MWh}$ on average, while the positive errors were penalized by only $10.29 € / \mathrm{MWh}$, and the model learned that the penalty was usually lower for positive errors. It seems that the second approach learned that this tendency of market prices was reliable enough to include it in the bidding behavior and thus systematically proposed lower bids, at the price of increasing the total imbalance. This 


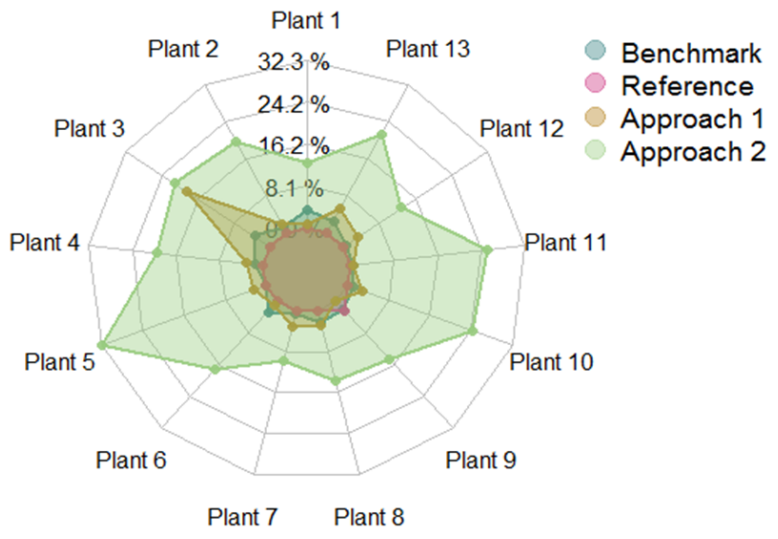

(a) Total imbalance

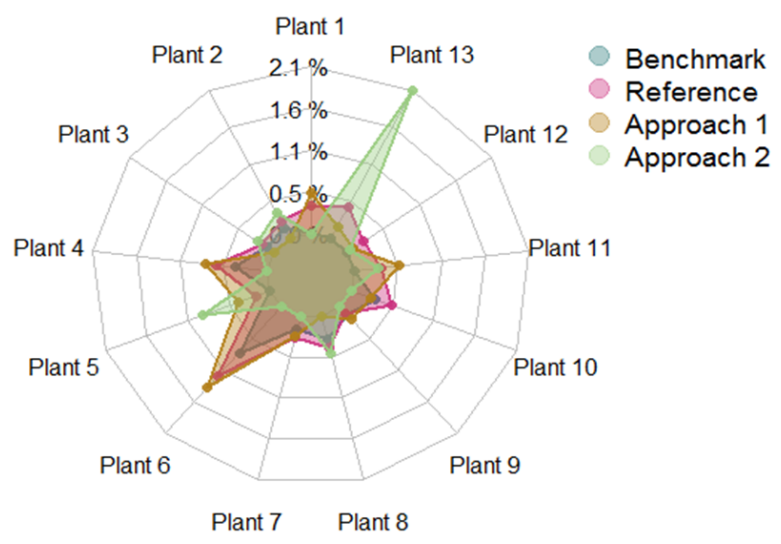

(b) Net revenue

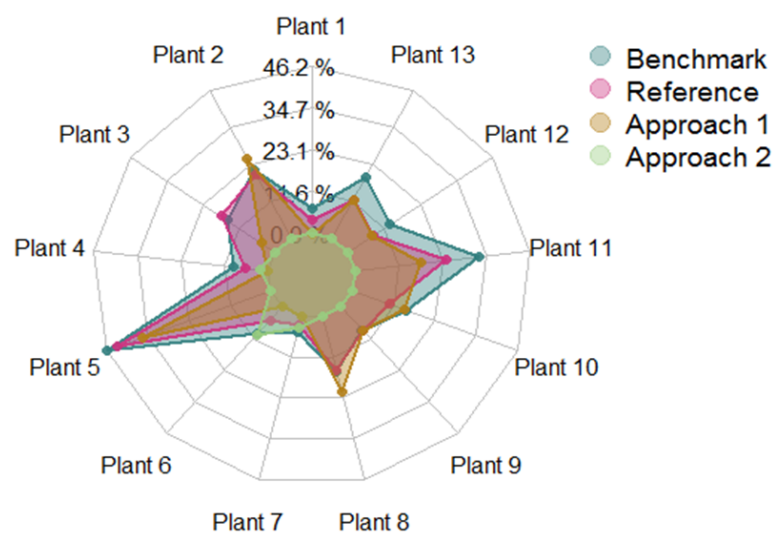

(c) Average penalty per imbalance

Fig. 6. Comparison of the different approaches

approach has proved effective with numerous power plants.

TABLE III

Average Bias of THE DIFFERENT APPROACHES

\begin{tabular}{ccccc}
\hline \hline & Bench. & Ref. & App. 1 & App 2 \\
\hline Bias (\%) & 0.098 & 0.33 & 0.096 & -0.82 \\
\hline \hline
\end{tabular}

Another effect can also be identified when looking at the evaluation results. We looked at the spread of the errors depending on the magnitude of the regulation prices for both positive and negative imbalances, in order to understand how the second approach could generate lower penalties with higher imbalances. The results are shown on Fig. 7 for plant 2 , and indicate that the errors in the second approach are concentrated on low penalty levels, especially for positive errors.

Overall, the models adapt in different ways to minimize revenue. Approach 1 tends to recalibrate the bidding process after incorporating market information. The second approach behaves differently, as it tends to offer lower bids than the expected production. This is because negative errors are usually penalized more heavily than positive ones. It is also much better at identifying instants when the regulation prices are high, with most of its errors occurring when the regulation price is low. However, since it does not rely on PV power forecasts, it creates more imbalances than the first approach.

The choice of a given approach depends highly on the context. The difference in revenue is not significant and should not be a sufficient incentive for the energy producer to choose one approach instead of another. However, the behavior of the approaches might be relevant. In a system where the difference between high and low balancing prices is significant, the second approach would be preferable, since it concentrates the imbalances when the balancing price is low. On the other hand, when this difference is low, any of the other approaches would be more beneficial since they generate fewer imbalances overall. If the regulating prices correctly translate the most preferable approach for the grid, the amount of penalties would be a sufficient criterion to decide on the best approach, because in that case, the approach that is the most supportive of the grid would also be subject to fewer penalties.

\section{Test CASe II: Trading of Wind Power on the NORDPOOL MARKET}

In this test case, we study the participation of a wind producer in the alternative electricity market NordPool. This test case is intended to illustrate the case of an RES producer that does not have the means to produce its own forecast, and thus buys forecasts from a third party. The goal is to show that even with limited input, the second approach can improve the decision-making process.

All data relative to the wind power come from a previous study [18]. The forecasts are obtained using regression forests and are deterministic. Therefore, in the following methodology, the CDF of the wind power forecasts $F_{w}$ are considered to be a Heaviside function on the deterministic forecast $E_{w}$ :

$$
F_{W}(p)=1 \text { if } p>E_{W}, 0 \text { otherwise }
$$

Note that in this case, market quantity forecasts cannot be used since they are employed to derive an optimal quantile of the $\mathrm{CDF}$ to bid on the electricity market, which will always reduce to the deterministic forecast of the wind power.

Besides, since we do not dispose of our own forecast model, we cannot apply the reference and first approaches to the trading problem, since they require optimization of the forecast model with different objective functions. Thus, only two strategies can be evaluated: the benchmark strategy, where the deterministic wind power forecast is bid on the electricity 


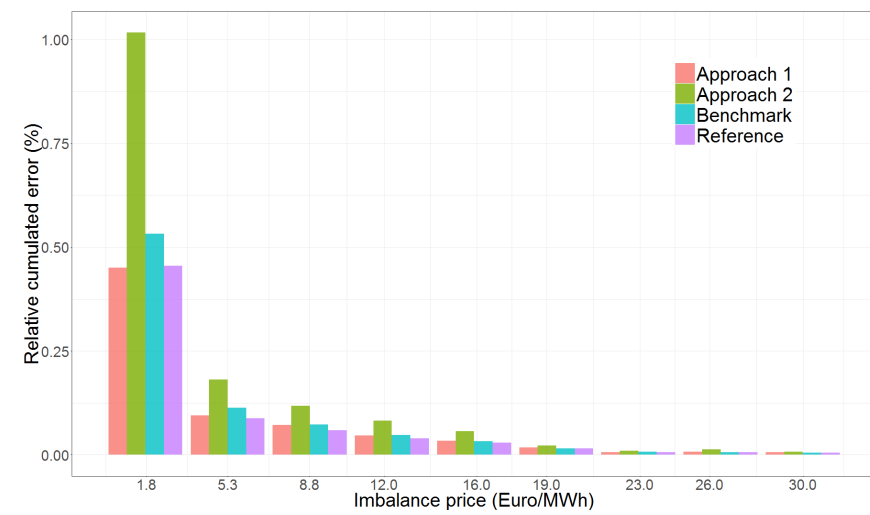

(a) Positive errors

Fig. 7. Cumulated errors depending on regulation price magnitude

market, and the second approach, where the deterministic wind power forecasts are used as inputs for the ELM model.

We study the output from nine wind power plants located in Denmark. In situ measurements of energy generation are available from January 2008 to October 2009. The year 2008 was used as the training period, and the period from January to October 2009 as the test period.

The benchmark approach is implemented by bidding the deterministic wind power forecasts. The second approach could also be implemented by using the wind power forecasts as input of the ELM model. Results from the two approaches are summarized in Table IV. In this study, once again, the second approach consistently produces more imbalances, however it almost always outperforms the benchmark.

This case study is of great value, as it consolidates the fact that the second approach produces bids that cause more imbalances, but lowers the average amount of penalties paid for any imbalance. In the first case study, this did not generate higher revenue, however, in the different electricity market involved in case study II, the revenue was higher with the second approach.

\section{CONCLUSiOnS}

In this paper, we introduced two novel approaches to deal with complex model chains that integrate forecasting and decision-making functions. The application example was that of trading RES production on a day-ahead market. The model chain in this case includes four forecasting models and one decision-making model. While the first approach can be perceived as a fairly straightforward extension of the metaoptimization currently employed in forecasting approaches, the second approach introduces a completely new paradigm since it replaces the initial model chain with a single, direct datadriven model. The contributions of the paper for this specific application are (1) the direct use of revenue as an objective function to optimize the parameters of the models, when using separate models for PV power forecasts and market quantity forecasts and (2) the proposal of a trading model that bypasses the models for PV and market quantity forecasting by using a neural network trained for market performance to directly produce bids.

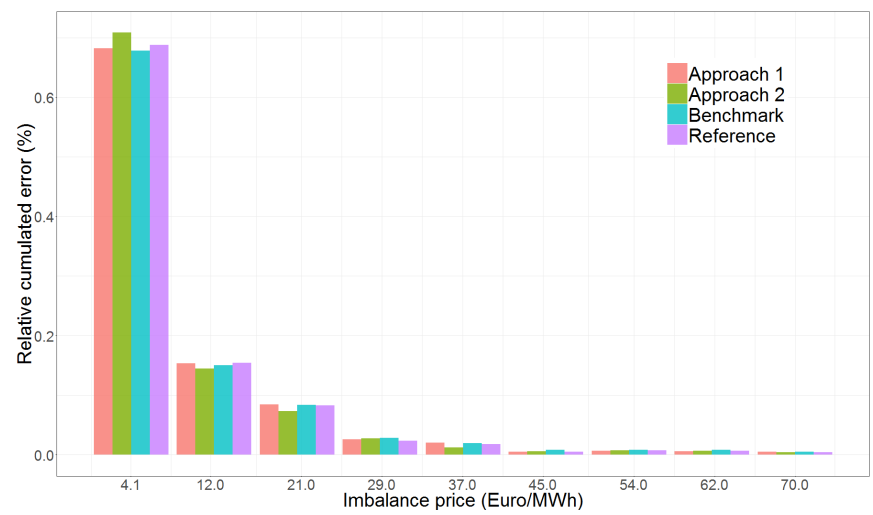

(b) Negative errors

All of the models behaved differently when using market performance as the objective function for optimizing their parameters. The first approach featuring separate models modified the individual models to recalibrate the bidding process after introducing market information, while the second approach acquired a negative bias to compensate the higher price of negative errors. The second approach also learned to minimize the errors when the price penalizing the imbalances was significant.

The approaches were implemented in two different case studies, one for trading PV power on the EPEX SPOT electricity market, and one for trading wind power on the NordPool market. In the first case, using the revenue as the objective function did not clearly create models that generate more revenue on electricity markets than the reference state-ofthe-art approach, although it was possible to identify bidding behavior trends in the different approaches. Compared to the first approach, the second approach is more efficient for trading, with a lower amount of penalty per imbalance, but it creates more imbalances. In the second case however, using the revenue as the objective yielded better results for all wind power plants but one. A research direction could be to use the uncertainty in the energy price with probabilistic forecasts and Monte-Carlo methods to improve further the effectiveness of using revenue as an objective function.

This paper illustrates the feasibility of a concept that opens broad perspectives for application in other functions related to power systems management. Further work could test this concept on energy markets with different pricing rules (i.e. single-pricing for imbalance settlement), to evaluate whether it is suitable for any set of market rules. This would require modifying the objective function of the optimization loops to correspond to market rules. In addition, in markets where several consecutive decisions have to be made (e.g. intra-day market sessions or control of a storage system coupled with the power plant), each decision-making process would have to be modeled with an ELM model. The authors expect that the best approach for a given application is primarily driven by the imbalance penalization rules, and extending the work to new market structures could test this hypothesis, as in our study no model clearly outperformed the others in dual-pricing 
TABLE IV

Evaluation Results for the Testing Period (JAN 2009 - OCtober 2009)

\begin{tabular}{ccccccccccc}
\hline \hline & Plant number & 1 & 2 & 3 & 4 & 5 & 6 & 7 & 8 \\
\hline & Forecast RMSE (\%) & 14.0 & 15.6 & 15.0 & 16.6 & 16.3 & 19.0 & 16.5 & 15.6 & 16.1 \\
Benchmark & Absolute Bids Error (MWh) & 654 & 746 & 705 & 785 & 764 & 946 & 774 & 752 & 757 \\
& Penalties (DKK) & 14969 & 17896 & 15303 & 17279 & 18681 & 22711 & 19055 & 15473 & 17082 \\
& Net Revenue (DKK) & 334704 & 379988 & 323179 & 367804 & 365797 & 438805 & 358754 & 386620 & 352124 \\
& Penalty per imbalance (DKK/MWh) & 22.4 & 22.9 & 21.2 & 22.2 & 23.8 & 23.2 & 23.2 & 21.6 & 22.3 \\
\hline & Forecast RMSE (\%) & - & - & - & - & - & - & - & - \\
Approach 2 & Absolute Bids Error (MWh) & 657 & 775 & 720 & 757 & 797 & 970 & 814 & 751 & 762 \\
& Penalties (DKK) & 14317 & 17645 & 14874 & 16841 & 18784 & 21043 & 18322 & 15193 & 17279 \\
& Net Revenue (DKK) & 335357 & 380239 & 323608 & 368242 & 365695 & 440473 & 359487 & 386900 & 351928 \\
& Penalty per imbalance (DKK/MWh) & 21.8 & 22.8 & 20.7 & 22.3 & 23.6 & 21.7 & 22.5 & 20.2 & 22.7 \\
\hline \hline
\end{tabular}

markets.

\section{ACKNOWLEDGMENTS}

The authors want to thank the company Solaïs for providing power and irradiation measurements from their PV plants. The authors would also like to thank the ECMWF for providing weather forecast data.

\section{REFERENCES}

[1] G. Kariniotakis, Renewable Energy Forecasting: From Models to Applications. Woodhead Publishing, 2017.

[2] P. McSharry, R. Girard, and P. Pinson, "Methodology for the evaluation of probabilistic forecasts",' Deliverable Dp-6.2, European (FP7) project SafeWind, 2009, Tech. Rep., 2007.

[3] R. Bessa, C. Möhrlen, V. Fundel, M. Siefert, J. Browell, S. Haglund El Gaidi, B.-M. Hodge, U. Cali, and G. Kariniotakis, "Towards Improved Understanding of the Applicability of Uncertainty Forecasts in the Electric Power Industry," Energies, vol. 10, no. 9, p. 1402, 2017.

[4] R. J. Bessa, V. Miranda, A. Botterud, and J. Wang, "good or bad wind power forecasts: a relative concept," Wind Energy, vol. 14, no. 5, pp. 625-636, 2011.

[5] A. Botterud, Z. Zhou, J. Wang, R. J. Bessa, H. Keko, J. Sumaili, and V. Miranda, "Wind power trading under uncertainty in LMP markets," IEEE Transactions on Power Systems, vol. 27, no. 2, pp. 894-903, 2012.

[6] A. Bracale, G. Carpinelli, P. De Falco, R. Rizzo, and A. Russo, "New advanced method and cost-based indices applied to probabilistic forecasting of photovoltaic generation," Journal of Renewable and Sustainable Energy, vol. 8, no. 2, 2016.

[7] R. Girard, K. Laquaine, and G. Kariniotakis, "Assessment of wind power predictability as a decision factor in the investment phase of wind farms," Applied Energy, vol. 101, pp. 609-617, 2013.

[8] A. Skajaa, K. Edlund, and J. M. Morales, "Intraday Trading of Wind Energy," IEEE Transactions on Power Systems, vol. 30, no. 6, 2015.

[9] A. Núñez-Reyes, D. Marcos Rodríguez, C. Bordons Alba, and M. Á. Ridao Carlini, "Optimal scheduling of grid-connected PV plants with energy storage for integration in the electricity market," Solar Energy, vol. 144 , pp. 502-516, 2017.

[10] N. Mazzi and P. Pinson, "Purely data-driven approaches to trading of renewable energy generation," in International Conference on the European Energy Market, vol. 2016-July, Porto, Portugal, 2016.

[11] H. T. C. Pedro and C. F. M. Coimbra, "Assessment of forecasting techniques for solar power production with no exogenous inputs," Solar Energy, vol. 86, no. 7, pp. 2017-2028, 2017.

[12] F. Golestaneh, P. Pinson, and H. B. Gooi, "Very Short-Term Nonparametric Probabilistic Forecasting of Renewable Energy Generation With Application to Solar Energy," IEEE Transactions on Power Systems, vol. 31, no. 5, 2016.

[13] S. Panda and N. P. Padhy, "Comparison of particle swarm optimization and genetic algorithm for FACTS-based controller design," Applied Soft Computing Journal, vol. 8, no. 4, pp. 1418-1427, 2008.

[14] S. Alessandrini, L. Delle Monache, S. Sperati, and G. Cervone, "An analog ensemble for short-term probabilistic solar power forecast," Applied Energy, vol. 157, pp. 95-110, 2015.
[15] G. Cervone, L. Clemente-Harding, S. Alessandrini, and L. Delle Monache, "Short-term photovoltaic power forecasting using Artificial Neural Networks and an Analog Ensemble," Renewable Energy, vol. 108, pp. 274-286, 2017.

[16] L. Gigoni, A. Betti, E. Crisostomi, A. Franco, M. Tucci, F. Bizzarri, and D. Mucci, "Day-Ahead Hourly Forecasting of Power Generation from Photovoltaic Plants," IEEE Transactions on Sustainable Energy, vol. 9 , no. 2, pp. 831-842, 2018.

[17] G. Biau and L. Devroye, Lectures on the Nearest Neighbor Method, 2015.

[18] R. Girard, K. Laquaine, and G. Kariniotakis, "Assessment of wind power predictability as a decision factor in the investment phase of wind farms," Applied Energy, vol. 101, pp. 609 - 617, 2013.

[19] D. Meyer, E. Dimitriadou, K. Hornik, A. Weingessel, and F. Leisch, e1071: Misc Functions of the Department of Statistics, Probability Theory Group (Formerly: E1071), TU Wien, 2017, r package version 1.6-8.

[20] C. Bendtsen., pso: Particle Swarm Optimization, 2012, r package version 1.0.3.

[21] P. Pinson, C. Chevallier, and G. N. Kariniotakis, "Trading wind generation from short-term probabilistic forecasts of wind power," IEEE Transactions on Power Systems, vol. 22, no. 3, pp. 1148-1156, 2007.

[22] E. Bitar, R. Rajagopal, and P. Khargonekar, "Bringing Wind Energy to Market," IEEE Transactions on Power Systems, 2011.

[23] M. Zugno, T. Jonnsson, and P. Pinson, "Trading wind energy on the basis of probabilistic forecasts both of wind generation and of market quantities," Wind Energy, 2013.

[24] R. Weron, "Electricity price forecasting: A review of the state-of-theart with a look into the future," International Journal of Forecasting, vol. 30, no. 4, pp. 1030-1081, 2014.

[25] S. Camal, A. Michiorri, and G. Kariniotakis, "Optimal Offer of Automatic Frequency Restoration Reserve from a Combined PV/Wind Virtual Power Plant," IEEE Transactions on Power Systems, vol. 99, no. c, p. 1, 2018.

[26] G. B. Huang, Q. Y. Zhu, and C. K. Siew, "Extreme learning machine: Theory and applications," Neurocomputing, vol. 70, no. 1-3, pp. 489$501,2006$.

[27] C. Wan, J. Lin, Y. Song, Z. Xu, and G. Yang, "Probabilistic Forecasting of Photovoltaic Generation: An Efficient Statistical Approach," IEEE Transactions on Power Systems, vol. 32, no. 3, pp. 2471-2472, 2017.

[28] T. Carriere, C. Vernay, S. Pitaval, F. Neirac, and G. Kariniotakis, "A novel approach for probabilistic photovoltaic power forecasting covering multiple time frames," submitted in IEEE Transactions on Smart Grid.

[29] T. Hastie, R. Tibshirani, and J. Friedman, The Elements of Statistical Learning, 2001, vol. 1.

[30] H. Peng, F. Long, and C. Ding, "Feature selection based on mutual information: Criteria of Max-Dependency, Max-Relevance, and MinRedundancy," IEEE Trans. on Pattern Analysis and Machine Intelligence, vol. 27, no. 8, pp. 1226-1238, 2005.

[31] M. Lefèvre, A. Oumbe, P. Blanc et al., "McClear: A new model estimating downwelling solar radiation at ground level in clear-sky conditions," Atmospheric Measurement Techniques, vol. 6, no. 9, pp. 2403-2418, 2013. 
ANNEX A: COMPLETE EVALUATION RESULTS OF THE FIRST TEST CASE

\begin{tabular}{|c|c|c|c|c|c|}
\hline Plant & & Benchmark & Reference & Approach 1 & Approach 2 \\
\hline & Price forecasts RMSE (\%) & - & 48.19 & 66.93 & - \\
\hline & AnEn forecasts CRPS (\%) & 5.81 & 5.81 & 5.89 & - \\
\hline \multirow{4}{*}{$1: 9828 \mathrm{kWp}$} & Absolute bids error (MWh) & 3116 & 3009 & 3033 & 3386 \\
\hline & Penalties (Eur) & 22171 & 20762 & 20143 & 22554 \\
\hline & Net revenue (Eur) & 405302 & 406711 & 407330 & 405245 \\
\hline & Average penalty per imbalance (Eur/MWh) & 7.11 & 6.90 & 6.64 & 6.66 \\
\hline \multirow{5}{*}{$2: 2694 \mathrm{kWp}$} & AnEn forecasts CRPS (\%) & 5.64 & 5.64 & 5.78 & - \\
\hline & Absolute bids error (MWh) & 834 & 823 & 839 & 987 \\
\hline & Penalties (Eur) & 5402 & 5270 & 5597 & 5267 \\
\hline & Net revenue (Eur) & 131209 & 131342 & 131015 & 131495 \\
\hline & Average penalty per imbalance (Eur/MWh) & 6.47 & 6.41 & 6.67 & 5.34 \\
\hline & AnEn forecasts CRPS (\%) & 4.79 & 4.79 & 6.01 & 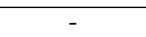 \\
\hline \multirow{4}{*}{$3: 10008.96 \mathrm{kWp}$} & Absolute bids error (MWh) & 2116 & 2043 & 2426 & 2478 \\
\hline & Penalties (Eur) & 13675 & 13441 & 14208 & 13881 \\
\hline & Net revenue (Eur) & 420860 & 421094 & 420327 & 421336 \\
\hline & Average penalty per imbalance (Eur/MWh) & 6.46 & 6.58 & 5.86 & 5.60 \\
\hline & AnEn forecasts CRPS (\%) & 5.25 & 5.25 & 5.38 & - \\
\hline \multirow[t]{4}{*}{$4: 6876 \mathrm{kWp}$} & Absolute bids error (MWh) & 2016 & 1984 & 2048 & 2375 \\
\hline & Penalties (Eur) & 14494 & 13829 & 13449 & 15920 \\
\hline & Net revenue (Eur) & 298524 & 299189 & 299569 & 297351 \\
\hline & Average penalty per imbalance (Eur/MWh) & 7.19 & 6.97 & 6.57 & 6.70 \\
\hline \multirow{5}{*}{$5: 4296 \mathrm{kWp}$} & AnEn forecasts CRPS $(\%)$ & 9.55 & 9.55 & 10.25 & - \\
\hline & Absolute bids error (MWh) & 2255 & 2253 & 2308 & 2981 \\
\hline & Penalties (Eur) & 14057 & 13754 & 13376 & 12692 \\
\hline & Net revenue (Eur) & 164241 & 164544 & 164922 & 165654 \\
\hline & Average penalty per imbalance (Eur/MWh) & 6.23 & 6.10 & 5.80 & 4.26 \\
\hline \multirow{5}{*}{$6: 11994 \mathrm{kWp}$} & AnEn forecasts CRPS (\%) & 5.91 & 5.91 & 6.01 & - \\
\hline & Absolute bids error (MWh) & 3996 & 3893 & 3929 & 4576 \\
\hline & Penalties (Eur) & 29246 & 27159 & 26172 & 33526 \\
\hline & Net revenue (Eur) & 524398 & 526485 & 527472 & 520387 \\
\hline & Average penalty per imbalance (Eur/MWh) & 7.32 & 6.98 & 6.66 & 7.33 \\
\hline \multirow{5}{*}{$7: 5064 \mathrm{kWp}$} & AnEn forecasts CRPS (\%) & 5.16 & 5.16 & 5.30 & - \\
\hline & Absolute bids error (MWh) & 1456 & 1447 & 1492 & 1590 \\
\hline & Penalties (Eur) & 8979 & 8775 & 8815 & 9722 \\
\hline & Net revenue (Eur) & 207045 & 207249 & 207209 & 206692 \\
\hline & Average penalty per imbalance (Eur/MWh) & 6.17 & 6.06 & 5.91 & 6.11 \\
\hline \multirow{5}{*}{$8: 9504 \mathrm{kWp}$} & AnEn forecasts CRPS (\%) & 5.58 & 5.58 & 5.74 & - \\
\hline & Absolute bids error (MWh) & 2922 & 2859 & 2941 & 3250 \\
\hline & Penalties (Eur) & 18194 & 17763 & 19238 & 17494 \\
\hline & Net revenue (Eur) & 364464 & 364896 & 363420 & 365180 \\
\hline & Average penalty per imbalance (Eur/MWh) & 6.23 & 6.21 & 6.54 & 5.38 \\
\hline \multirow{5}{*}{$9: 9504 \mathrm{kWp}$} & AnEn forecasts CRPS (\%) & 5.67 & 5.67 & 5.63 & - \\
\hline & Absolute bids error (MWh) & 2831 & 2839 & 2773 & 3184 \\
\hline & Penalties (Eur) & 18290 & 18367 & 17974 & 18964 \\
\hline & Net revenue (Eur) & 377056 & 376979 & 377372 & 376591 \\
\hline & Average penalty per imbalance (Eur/MWh) & 6.46 & 6.47 & 6.48 & 5.96 \\
\hline \multirow{5}{*}{$10: 4224 \mathrm{kWp}$} & AnEn forecasts CRPS (\%) & 5.45 & 5.45 & 5.64 & - \\
\hline & Absolute bids error (MWh) & 1258 & 1245 & 1282 & 1551 \\
\hline & Penalties (Eur) & 7570 & 7190 & 7665 & 8111 \\
\hline & Net revenue (Eur) & 179434 & 179815 & 179339 & 178894 \\
\hline & Average penalty per imbalance (Eur/MWh) & 6.02 & 5.77 & 5.98 & 5.23 \\
\hline \multirow{5}{*}{$11: 3300 \mathrm{kWp}$} & AnEn forecasts CRPS $(\%)$ & 5.00 & 5.00 & 5.08 & - \\
\hline & Absolute bids error (MWh) & 889 & 884 & 889 & 1109 \\
\hline & Penalties (Eur) & 6015 & 5597 & 5328 & 5656 \\
\hline & Net revenue (Eur) & 127122 & 127540 & 127809 & 127496 \\
\hline & Average penalty per imbalance (Eur/MWh) & 6.77 & 6.33 & 5.99 & 5.10 \\
\hline \multirow{5}{*}{$12: 2610 \mathrm{kWp}$} & AnEn forecasts CRPS $(\%)$ & 5.16 & 5.16 & 5.33 & - \\
\hline & Absolute bids error (MWh) & 743 & 739 & 764 & 836 \\
\hline & Penalties (Eur) & 4690 & 4449 & 4597 & 4666 \\
\hline & Net revenue (Eur) & 98424 & 98665 & 98517 & 98471 \\
\hline & Average penalty per imbalance (Eur/MWh) & 6.32 & 6.02 & 6.01 & 5.58 \\
\hline \multirow{5}{*}{$13: 11994 \mathrm{kWp}$} & AnEn forecasts CRPS (\%) & 5.18 & 5.18 & 5.38 & - \\
\hline & Absolute bids error (MWh) & 3084 & 3009 & 3170 & 3656 \\
\hline & Penalties (Eur) & 22173 & 20414 & 21493 & 22095 \\
\hline & Net revenue (Eur) & 382414 & 384173 & 383094 & 390523 \\
\hline & Average penalty per imbalance (Eur/MWh) & 7.19 & 6.78 & 6.78 & 6.04 \\
\hline
\end{tabular}


Thomas Carriere is graduated from Ecole Supélec (2016) with a specialization in Energetics, and received is M.S. degree in Energy Processes from the Universite Paris-Saclay (2016). He is pursuing a Ph.D. degree at MINES ParisTech, PSL - Research University, PERSEE - Centre for Processes, Renewable Energies and Energy Systems. His research interests are forecasting from statistical modelling, storage systems and renewable energies integration to electricity markets.

Georges Kariniotakis (S'95-M'02-SM'11) was born in Athens, Greece. He received his Eng. and M.Sc. degrees from Greece in 1990 and 1992 respectively, and his PhD from Ecole des Mines de Paris in 1996. He currently works at the MINES ParisTech PERSEE Centre as a senior scientist and head of the Renewable Energies and Smart Grids Group. He has authored more than 220 scientific publications in journals and conferences. He has been involved as participant or coordinator in more than $40 \mathrm{R} \& \mathrm{D}$ projects in the fields of renewable energies and distributed generation. Among them, he was the coordinator of some major EU projects in the field of wind power forecasting such as Anemos, Anemos.plus and SafeWind projects. His scientific interests include among others timeseries forecasting, decision making under uncertainty, modeling, management and planning of power systems. 